\title{
Thiolated chitosan nanoparticles for enhancing oral absorption of docetaxel: preparation, in vitro and ex vivo evaluation
}

This article was published in the following Dove Press journal:

International Journal of Nanomedicine

II January 20 I I

Number of times this article has been viewed

\author{
Shahrooz Saremi ${ }^{1,4}$ \\ Fatemeh Atyabi ${ }^{1,2}$ \\ Seyedeh Parinaz Akhlaghi' \\ Seyed Nasser Ostad ${ }^{3}$ \\ Rassoul Dinarvand ${ }^{1,2}$ \\ 'Department of Pharmaceutics, \\ ${ }^{2}$ Nanotechnology Research Centre, \\ ${ }^{3}$ Department of Toxicology and \\ Pharmacology, Faculty of Pharmacy, \\ Tehran University of Medical Sciences, \\ Tehran, Iran; ${ }^{4}$ R\&D Department, \\ Osvah Pharmaceutical Co., \\ Tehran, Iran
}

\begin{abstract}
The aim of this study was to prepare and evaluate mucoadhesive core-shell nanoparticles based on copolymerization of thiolated chitosan coated on poly methyl methacrylate cores as a carrier for oral delivery of docetaxel. Docetaxel-loaded nanoparticles with various concentrations were prepared via a radical emulsion polymerization method using cerium ammonium nitrate as an initiator. The physicochemical properties of the obtained nanoparticles were characterized by: dynamic light-scattering analysis for their mean size, size distribution, and zeta potential; scanning electron microscopy and transmission electron microscopy for surface morphology; and differential scanning calorimetry analysis for confirmation of molecular dispersity of docetaxel in the nanoparticles. Nanoparticles were spherical with mean diameter below $200 \mathrm{~nm}$, polydispersity of below 0.15 , and positive zeta potential values. The entrapment efficiency of the nanoparticles was approximately $90 \%$. In vitro release studies showed a sustained release characteristic for 10 days after a burst release at the beginning. Ex vivo studies showed a significant increase in the transportation of docetaxel from intestinal membrane of rat when formulated as nanoparticles. Cellular uptake of nanoparticles was investigated using fluoresceinamine-loaded nanoparticles. Docetaxel nanoparticles showed a high cytotoxicity effect in the Caco- 2 and MCF-7 cell lines after 72 hours. It can be concluded that by combining the advantages of both thiolated polymers and colloidal particles, these nanoparticles can be proposed as a drug carrier system for mucosal delivery of hydrophobic drugs.
\end{abstract}

Keywords: poly methyl methacrylate, mucoadhesive, cytotoxicity

\section{Introduction}

Oral drug delivery is the most acceptable method of drug delivery to the body. It is particularly important for cytotoxic medicines. These drugs are generally available as injectable dosage forms and their administration requires experienced personnel, patient's presence at the hospital, and specific care, and thus poses many problems for the patients. Some of the advantages of oral chemotherapy include: increasing the drug efficacy and half-life, preventing excess increase in the blood concentration and the increase of drug toxicity, as well as decreasing the drug side effects in the body. ${ }^{1,2}$ For these reasons, many studies have been carried out in recent years on the administration of cytotoxic drugs in oral dosage form..$^{2-6}$ Docetaxel (DTX) is a semisynthetic taxoid extract from Taxus baccata (European yew tree) and is one of the most effective drugs in chemotherapy. ${ }^{7}$ The mechanism of this drug on cancer cells is through inhibiting microtubule depolymerization, and studies show that the cytotoxic effect of DTX on tumor cells is about two times that of paclitaxel. ${ }^{8}$ DTX is used as an effective drug against breast, ovarian, lung, head, and neck cancers ${ }^{9-11}$ and is available only
Correspondence: Fatemeh Atyabi Faculty of Pharmacy, Tehran University of Medical Sciences,

Tehran, PO Box I4I55-645I, Iran

$\mathrm{Tel}+982166959052$

Fax +98 21 66959052

Email atyabifa@tums.ac.ir 
in the parenteral dosage form (Taxotere; ${ }^{\circledR}$ sanofi-aventis). Because of poor solubility of DTX in water, it is formulated as a solution with a high amount of Tween 80/ethanol. High concentration of solubilizers in its formula causes toxic effects and allergic reactions. ${ }^{12}$ Like most effective drugs used in chemotherapy, it is not absorbable orally and has a very low bioavailability. In addition to its poor aqueous solubility, it is eliminated through the first pass extraction by the cytochrome $\mathrm{P}-450$ processes and the action of efflux pump of p-glycoproteins (p-gp) in the liver and intestine. ${ }^{13,14}$ The use of surface-modified polymeric nanoparticle (NP) systems may be regarded as an effective means of overcoming these problems. NPs, due to their unique properties such as their small size and certain surface characteristics, can protect drugs from $\mathrm{p}$-gp and cytochrome $\mathrm{P} 450$, protect drugs from the destructive factors in the gastrointestinal tract, and increase their permeability through the gastrointestinal barrier. ${ }^{2}$ Considering the nature of these NPs, an interesting aspect in preparing surface-modified NPs is based on the synthesis of amphiphilic copolymers. ${ }^{5,15-18}$ Synthesizing copolymers using polyalkylcyanoacrylate and a polysaccharide such as chitosan or dextran, as described by Chauvierre et al, ${ }^{18}$ is a very favorable approach due to the simultaneous formation of NP and copolymer in a single stage. Here, NP formation is by a radical emulsion polymerization method, which is initiated by the creation of a free radical at the end of the polysaccharide chain, through an initiator like cerium ion in acidic medium; these free radicals cause the polymerization of cyanoacrylate monomers and the formation of a linear block copolymer. Since this reaction is carried out in an aqueous medium, the hydrophobic polymer tends to go inside the NPs at the same time that the particle is formed, and creates a hydrophobic core coated with a hydrophilic polysaccharide. This method results in the formation of a very stable NP suspension. ${ }^{17-19}$

One of the polymers which has attracted much interest is chitosan, due to its excellent characteristics for preparing surface-modified NPs. Chitosan is a nontoxic, biocompatible, and biodegradable polymer that is able to bind to mucus due to ionic interactions between its primary amino substructures and the sialic acid and sulfonic acid substructures of the mucus. $^{20}$

The mucoadhesive properties of chitosan are increased intensively by the immobilization of thiol groups on its structure. $^{21}$ The mucoadhesive properties of chitosan have been shown to improve 140 -fold due to the immobilization of thiol groups on the polymer. ${ }^{21}$ The reason for this is the formation of disulfide bonds between the thiolated polymer and cysteine-rich subdomains of the mucus gel layer. ${ }^{22}$
The thiolated derivatives cause an increase in permeability through the intestine membrane by the mechanism of the regeneration of glutathione and inactivation of the protease enzymes in the gastrointestinal tract via absorbing and removing the bivalent cations from the medium. ${ }^{6,23}$ All these properties make the thiolated chitosan a very desirable material for increasing the absorption of peptides and NPs through the mucosa. ${ }^{24}$ Due to these favorable characteristics of chitosan, many researchers in recent years have investigated the preparation and evaluation of NPs obtained from the copolymerization of acrylate derivatives, coated with chitosan or its derivatives. ${ }^{17-19,25}$

In this study, the potential use of NPs prepared by the copolymerization of poly methyl methacrylate (pMMA) and chitosan-glutathione conjugates as a carrier for oral delivery of a hydrophobic drug (DTX) was investigated.

\section{Materials and methods \\ Materials}

DTX was obtained from Cipla (Mumbai, India). Chitosan (ChitoClear) with a medium molecular weight and degree of deacetylation of about $89 \%$ was purchased from Primex (Karmoy, Norway). L-Glutathione-reduced form (GSH), 1-ethyl-3-(3-dimethylaminopropyl) carbodiimide (EDC) hydrochloride, N-hydroxysuccinimide (NHS), methyl methacrylate (MMA), ammonium cerium nitrate, sodium nitrite, hydrochloric acid, glacial acetic acid, sodium hydroxide, and potassium hydrogen phosphate were all purchased from Merck (Darmstadt, Germany). Fluoresceinamine (FA) isomer I and Ellman's reagent, 5,50-dithiobis (2-nitro benzoic acid), were obtained from Sigma-Aldrich (St. Louis, MO). 3-(4,5- dimethylthiazol-2yl)-2,5-diphenyl tetrazolium bromide) (MTT) was purchased from Sigma-Aldrich. Caco-2 and MCF-7 cell lines were obtained from the Pasteur Institute (Tehran, Iran). All other chemicals were of analytical grade.

\section{Depolymerization of chitosan}

Chitosan was selectively depolymerized following the method developed by Huang et al. ${ }^{26}$ Briefly, $100 \mathrm{~mL}$ of a $2 \%$ $(\mathrm{w} / \mathrm{v})$ chitosan $(400,000 \mathrm{~g} / \mathrm{mol})$ solution in $6 \%(\mathrm{v} / \mathrm{v})$ acetic acid solution was prepared. Then the dissolved chitosan was depolymerized at room temperature under stirring with $10 \mathrm{~mL}$ of $\mathrm{NaNO}_{2}$ solutions in water at different concentrations $(7.0,2.7$, and $1.6 \mathrm{~g} / \mathrm{L})$, to obtain the desired final molecular weight of 20,000, 50,000, and 100,000 g/mol, respectively. After 1 hour of reaction, precipitated chitosan was obtained by raising the $\mathrm{pH}$ to 9.0 by addition of $4 \mathrm{~N}$ 
$\mathrm{NaOH}$. The white-yellowish solid was filtrated, washed thoroughly with acetone, and re-dissolved in a minimum volume of acetic acid $0.1 \mathrm{~N}(40 \mathrm{~mL})$.

Purification was carried out by subsequent dialyses against purified water (Sigma dialysis tubes, molecular weight cutoff, $12 \mathrm{kDa}$ ). The dialyzed product was lyophilized using a LyoTrap plus Freeze dryer (LTE Scientific, Oldham, UK), and the yellowish lyophilized product was then stored at $4^{\circ} \mathrm{C}$ until use. Products obtained were called Chito.20, Chito.50, and Chito100 depending on their theoretical molecular weight.

The average molecular weights of the prepared chitosans were determined by a viscometric method as described in our previous work. ${ }^{5}$

\section{Chitosan thiolation}

Thiolated chitosan was prepared with covalent attachment of reduced glutathione to different molecular weight of chitosan according to the slightly modified method described by Atyabi et al and Kafedjiiski et al as follows:5,27

One gram of chitosan was dispersed in $50 \mathrm{~mL}$ demineralized water by stirring, then $10 \mathrm{~mL} \mathrm{HCl}(1 \mathrm{~mol} / \mathrm{L})$ was added and finally dissolved by the addition of demineralized water to obtain a $1 \%(\mathrm{w} / \mathrm{v})$ polymer solution. The $\mathrm{pH}$ was adjusted to 6.0 by the addition of $\mathrm{NaOH}(5 \mathrm{~mol} / \mathrm{L})$. Afterwards, $5 \mathrm{~g}$ of reduced glutathione in $10 \mathrm{~mL}$ demineralized water was added to the above solution under continuous stirring. EDC and NHS were then added in a final concentration of $200 \mathrm{mmol} / \mathrm{L}$ each. The $\mathrm{pH}$ was readjusted to 6.0 by adding $\mathrm{NaOH}(5 \mathrm{~mol} / \mathrm{L})$. The reaction mixture was incubated for 15 hours at room temperature under constant stirring. To eliminate unbound reagents, the resulting polymer conjugate was dialyzed using dialysis tubing (molecular weight cutoff $12 \mathrm{kDa}$ ), first against $5 \mathrm{mmol} / \mathrm{L} \mathrm{HCl}$, twice against $5 \mathrm{mmol} / \mathrm{L}$ $\mathrm{HCl}$ containing $1 \% \mathrm{NaCl}$, and finally twice against $1 \mathrm{mmol} / \mathrm{L}$ $\mathrm{HCl}$. Controls were prepared in the same way but omitting EDC and NHS during the coupling reaction. Finally, the frozen aqueous polymer solutions were lyophilized at $-50^{\circ} \mathrm{C}$ and 0.01 mbar (LyoTrap plus; LTE Scientific, UK) and stored at $4^{\circ} \mathrm{C}$ until further use.

The amount of thiol groups immobilized on chitosanGSH conjugate was determined using Ellman's reagent and the spectrophotometric method. ${ }^{27-29}$

\section{Preparation of thiolated chitosan-pMMA NPs}

The NPs were prepared by using a modified radical polymerization method. Conjugated chitosan $(37.5 \mathrm{mg})$ with different theoretical molecular weight $(20,50$, and $100 \mathrm{kDa})$ was dissolved in $4 \mathrm{~mL}$ nitric acid $(0.2 \mathrm{~mol} / \mathrm{L})$ in a two-necked flask at $40^{\circ} \mathrm{C}$, under gentle stirring and nitrogen bubbling. After 10 minutes, under vigorous magnetic stirring, a solution of $0.08 \mathrm{~mol} / \mathrm{L}$ cerium IV ammonium nitrate (CAN) in $0.2 \mathrm{~mol} / \mathrm{L}$ nitric acid and MMA was added to obtain a $5-\mathrm{mL}$ solution. Nitrogen bubbling was continued for an additional 10 minutes. The reaction was allowed to continue at $40^{\circ} \mathrm{C}$ under gentle stirring for 40 minutes. After cooling to room temperature, the $\mathrm{pH}$ of the obtained suspension was adjusted to 4.5 by addition of sodium hydroxide $(1 \mathrm{~N})$ drop-wise. The NP suspension was then purified by dialysis (Sigma dialysis tubes, molecular weight cutoff, $12 \mathrm{kDa}$ ) against $1 \mathrm{~L}$ of acetic acid solution $(16 \mu \mathrm{mol} / \mathrm{L})$ in demineralized water, twice for 90 minutes and once overnight. The frozen NP suspensions were lyophilized at $-50^{\circ} \mathrm{C}$ and $0.01 \mathrm{mbar}$ and stored at $4^{\circ} \mathrm{C}$ until further use.

\section{Determination of the thiol group content of thiolated chitosan-coated pMMA (Cht-GSH-pMMA) NPs}

To quantify thiol group content of the NPs, iodine titration was performed as described by Bravo-Osuna et al and Kast and Bernkop-Schnürch. ${ }^{24,30}$ Twenty milligrams of the lyophilized NPs was hydrated in $6 \mathrm{~mL}$ of demineralized water. Following adjustment of $\mathrm{pH}$ to $2-3$ with $1 \mathrm{~mol} / \mathrm{L} \mathrm{HCl}$, the solution became clear. Then $500 \mu \mathrm{L}$ of aqueous starch solution (1\%) was added. The samples were titrated with an aqueous iodine solution ( $1 \mathrm{mmol} / \mathrm{L})$ until a permanent light blue color was maintained.

\section{Preparation of DTX- or FA-loaded NPs}

The anticancer drug-loaded NPs were prepared using Chito.20. After adding acidic solution of CAN, DTX was dissolved in $0.5 \mathrm{~mL}$ of methanol under stirring, and $0.25 \mathrm{~mL}$ MMA was added to obtain a clear solution. Under vigorous magnetic stirring, a solution of DTX was added in a two-necked flask. Nitrogen bubbling was continued for an additional 10 minutes. The added amount of DTX was in the range from $1 \%$ to $4 \%(\mathrm{w} / \mathrm{w})$ based on the weight of MMA and thiolated chitosan. The reaction was allowed to continue at $40^{\circ} \mathrm{C}$ under gentle stirring for 40 minutes. After adjusting the $\mathrm{pH}$ of the obtained suspension to 4.5, the NP suspension was purified by dialysis. The amount of DTX in the obtained NPs was measured by high-performance liquid chromatography (HPLC). Isocratic reversed-phase HPLC was performed using a Knauer HPLC system (Knauer, Berlin, Germany) with a $5 \mu$ Bondapak C18 column (Waters, Milford, MA). 
The mobile phase consisted of 75:25 (v/v) methanol/water and was delivered at a flow rate of $1.0 \mathrm{~mL} / \mathrm{min}$. Eluted compounds were detected at $227 \mathrm{~nm}$ using a Spectra100 UV-Vis detector. The FA-loaded NPs were prepared in the same way except for the addition of $0.25 \%(\mathrm{w} / \mathrm{v}) \mathrm{FA}$ to the monomer (MMA) solution instead of DTX.

\section{Size distribution of NPs}

The mean diameter and size distribution of the NPs were determined by dynamic light scattering using Zetasizer ${ }^{\circledR}$ (Nano-ZS, Malvern, Instruments, Malvern, UK). All dynamic light scattering measurements were carried out at a wavelength of $633 \mathrm{~nm}$ at $25^{\circ} \mathrm{C}$ with an angle detection of $90^{\circ}$. The samples were diluted in acetic acid $(16 \mu \mathrm{mol} / \mathrm{L})$ in deionized water, three subsequent measurements were determined for each sample, and the result was expressed as mean size \pm standard deviation.

\section{Determination of zeta potential}

The zeta potential measurements were performed by Laser Doppler Electrophoresis using Zetasizer (Nano-ZS). To maintain a constant ionic strength, the samples were diluted $(1: 50 \mathrm{v} / \mathrm{v})$ in $\mathrm{NaCl} 1 \mathrm{mmol} / \mathrm{L}(\mathrm{pH} 6.5) .{ }^{3}$ Each sample was measured three times.

\section{Differential scanning calorimetry (DSC)}

DSC was performed to investigate the physical status of DTX in the NPs. DSC scans of DTX, empty and DTX-loaded NPs were performed on a Mettler DSC $823^{\mathrm{e}}$ equipped with Mettler STAR ${ }^{\mathrm{e}}$ system software for the data acquisition. The samples were scanned at a speed of $5^{\circ} \mathrm{C} / \mathrm{min}$ in a $30^{\circ} \mathrm{C}-250^{\circ} \mathrm{C}$ temperature range.

\section{Scanning electron microscopy}

The surface morphology of the NPs was evaluated by using a scanning electron microscope (SEM XL 30; Philips, Eindhoven, The Netherlands). NP suspensions were successively diluted in deionized water to $1 / 50(\mathrm{v} / \mathrm{v})$. The dilutions were spread on an aluminum disc and dried at room temperature before the analysis. The dried NPs were then coated with a thin layer of gold metal using a sputter coater (SCD 005; Bal-Tec, Pfaffikon, Switzerland).

\section{Transmission electron microscopy (TEM)}

Transmission electron microscopy (TEM; CEM 902A; Zeiss, Oberkochen, Germany) was used to examine the structure and the topography of the NPs. NP suspensions were diluted in demineralized water to $1 / 100(\mathrm{v} / \mathrm{v})$, and prior to observation, the NPs were negatively stained with a solution of $1 \%$ phosphotungstic acid.

\section{Drug loading and entrapment efficiency}

The entrapment efficiency (EE) of the process was determined indirectly upon separation of the NPs by ultracentrifugation at $25,000 \mathrm{rpm}, 8^{\circ} \mathrm{C}$ for 30 minutes from the aqueous medium containing free DTX. The amount of free DTX in the supernatant was measured using HPLC. The EE of DTX NPs was calculated as the ratio of DTX loaded into the NPs with respect to the total amount of DTX used for preparation of the original mixture as follows:

$$
\mathrm{EE}(\%)=\left(\mathrm{DTX}_{\mathrm{t}}-\mathrm{DTX}_{\mathrm{f}}\right) / \mathrm{DTX}_{\mathrm{t}} \times 100,
$$

where DTX $\mathrm{D}_{t}$ is the total amount of DTX used for preparation of the original mixture and DTX $_{f}$ is the free DTX amount recovered in the supernatant. All samples were measured in triplicate.

Drug loading (DL) was calculated as follows:

DL $(\%)=($ Weight of loaded drug/Weight of NPs $) \times 100$.

\section{In vitro drug release study}

Drug release from DTX-loaded NPs was studied by incubating the NPs in phosphate-buffered solution (PBS), at $\mathrm{pH} 7.4$, at $37^{\circ} \mathrm{C}$. Two milligrams of NPs was dispersed in $5 \mathrm{~mL}$ of release medium (PBS of $\mathrm{pH} 7.4$ containing $0.1 \% \mathrm{w} / \mathrm{v}$ Tween 80 ) in a dialysis tube (Sigma dialysis tubes, molecular weight cutoff, $12 \mathrm{kDa}$ ), and the closed dialysis bag immersed in $20 \mathrm{~mL}$ release medium in a centrifuge tube. Tween 80 was used to increase the solubility of DTX in the buffer solution to maintain sink condition. The tube was placed in a shaker bath at $37^{\circ} \mathrm{C}$ and shaken horizontally at 100 cycles $/ \mathrm{min}$. At given time intervals, $15-\mathrm{mL}$ samples were withdrawn and replaced with the same volume of fresh medium. The samples were filtered through a $0.22-\mu \mathrm{m}$ filter and were analyzed for the amount of DTX using HPLC.

\section{In vitro cytotoxicity of DTX-loaded NPs}

Human colon adenocarcinoma cells (Caco2) and MCF-7 cells were used for the evaluation of cell viability of DTXloaded NPs. Separately, Caco-2 and MCF-7 cells were incubated in 96-well transparent plates (Costar, Chicago, IL) at $1 \times 10^{4}$ cells/well $(100 \mu \mathrm{L})$. After 12 hours the old medium was removed, and the cells were incubated in the medium containing DTX or DTX-loaded NPs. Because of the cytotoxic effects of Tween $80,{ }^{4,31}$ DTX was dissolved in 
dimethyl sulfoxide (DMSO) instead of Tween 80/ethanol/ saline as solvent. DTX was dissolved in DMSO at a proper concentration and diluted 100-fold with growth medium. DTX-loaded NPs were then dispersed into the purified water, and after dilution with growth medium were added to each well at equivalent drug concentrations ranging from 0.1 to $1000 \mathrm{ng} / \mathrm{mL}$. After 72 hours incubation, $20 \mu \mathrm{L}$ of MTT (3-(4,5-dimethylthiazol-2-yl)-2, 5-diphenyl tetrazoniumbromide) solution $(5 \mathrm{mg} / \mathrm{mL})$ was added to each well of the plate. After 4 hours, $100 \mu \mathrm{L}$ DMSO was added to each well, and the absorbance of the solution was measured at $570 \mathrm{~nm}$ and reference wavelength at $690 \mathrm{~nm}$ by a microplate reader (Anthos 2020; Anthos Labtec Instruments, Wals, Austria).

\section{Caco- 2 cell uptake of fluorescent NPs}

For qualitative uptake studies, the Caco- 2 cells were seeded in the chambered glass system (Lab-Tek; Nunc International Co., Naperville, IL). Cells were washed four times after incubation with FA-loaded NPs for 2 hours and then fixed by a cold mixture of methanol/acetone $(50: 50 \mathrm{v} / \mathrm{v})$ for 15 minutes at room temperature. The cells were washed twice with PBS and mounted in mounting medium consisting of $\mathrm{Na}_{2} \mathrm{HPO}_{4}$ and acetic acid (pH 5.5)/glycerol (50:50 v/v) to be observed by fluorescence microscope ( $\lambda$ ext: $540 \mathrm{~nm}$ and $\lambda$ em: $580 \mathrm{~nm}$; BX40; Olympus, Tokyo, Japan). The fluorescent images were taken by DP70 digital imaging system (Olympus) and analyzed by Olysia imaging software (Olympus).

\section{Ex vivo study}

The everted sac method was chosen for measurement of transportation of DTX across the intestine barrier. It was carried out according to the slightly modified method that was described by Barr and Riegelman, ${ }^{32}$ as follows.

First, a section of about $5 \mathrm{~cm}$ of the jejunum was removed from a male rat under ketamine $(50 \mathrm{mg} / \mathrm{kg})$ and chlorpromazine $(10 \mathrm{mg} / \mathrm{kg})$ anesthesia and washed with Krebs-Ringer bicarbonate solution of $\mathrm{pH}=7.4$. This section was then gently inverted with a glass rod, and a tube was inserted in one side of the section and tied securely with tape. The other side of the intestine was tied, and $1 \mathrm{~mL}$ Krebs-Ringer bicarbonate solution was poured through the hypodermic needle in the tube. The gut sac was placed in a medium saturated with $95 \%$ $\mathrm{O}_{2}, 5 \% \mathrm{CO}_{2}$, and contained the test sample in Krebs-Ringer bicarbonate solution at $37^{\circ} \mathrm{C}$. The test samples used include: (1) DTX (1 mg) as Taxotere ${ }^{\circledR}$, and (2) Chito.20-GSH-DTX $4 \%$ NPs (equivalent to $1 \mathrm{mg}$ of DTX). In absorption studies, an $\mathrm{O}_{2}$ and $\mathrm{CO}_{2}$ mixture was bubbled into the intestinal mucosa to obtain intestinal peristaltic movement. At certain periods of time, $0.5-\mathrm{mL}$ samples were drawn from inside the intestine and replaced with the same volume of fresh medium. The amount of transported DTX in the samples was measured by the HPLC method.

\section{Results and discussion Chitosan depolymerization and characterization}

Several studies have demonstrated that the molecular weight of chitosan is very important to NP properties such as particle size, ${ }^{33}$ paracellular permeability, ${ }^{34}$ and immunological response. ${ }^{35}$ Due to high molecular weight and thus high viscosity and poor solubility at physiological $\mathrm{pH}$ range of the commercial chitosan, the use of low-molecular-weight chitosan is preferred in particular drug delivery systems. ${ }^{36}$

Depolymerization of chitosan was carried out via an oxidative process by $\mathrm{NaNO}_{2}$ as a simple and reproducible method. Molecular weights of depolymerized chitosan determined by capillary viscometry measurement are shown in Table 1. It has been demonstrated that various molecular weights of chitosans could be obtained by changing the chitosan $/ \mathrm{NaNO}_{2}$ molar ratio. ${ }^{37,38}$ As expected, using distinct amounts of $\mathrm{NaNO}_{2}$ produced chitosans with molecular weights in accordance with theoretical values. ${ }^{5,33}$

\section{Thiolated chitosan preparation and quantification}

Table 1 summarizes the amounts of free thiol groups, disulfide bonds, and total sulfhydryl groups immobilized on chitosan-GSH conjugates. The free thiol content and total

Table I Molecular weights of depolymerized chitosan and thiolated chitosan characterization $(n=3)$

\begin{tabular}{|c|c|c|c|c|c|}
\hline $\begin{array}{l}\text { Chitosan } \\
\text { type }\end{array}$ & $\begin{array}{l}\mathrm{NaNO}_{2} \\
\mathrm{mg} / \mathrm{mL}\end{array}$ & $\begin{array}{l}\text { Determined } \\
\text { Mw (g/mol) }\end{array}$ & $\begin{array}{l}\text { Free thiol } \\
(\mu \mathrm{mol} / \mathrm{g})\end{array}$ & $\begin{array}{l}\text { Disulfide content } \\
(\mu \mathrm{mol} / \mathrm{g})\end{array}$ & $\begin{array}{l}\text { Total amount of } \\
\text { sulfhydryl groups }(\mu \mathrm{mol} / \mathrm{g})\end{array}$ \\
\hline Chito.20 & 7.0 & 19,230 & $93.70 \pm 4.20$ & $552.66 \pm 12.14$ & $646.36 \pm 8.05$ \\
\hline Chito.50 & 2.7 & 53,850 & $86.68 \pm 5.70$ & $532.17 \pm 10.53$ & $618.85 \pm 5.61$ \\
\hline Chito. 100 & 1.6 & 97,520 & $69.11 \pm 4.72$ & $529.04 \pm 18.57$ & $598.15 \pm 23.31$ \\
\hline Parent & - & 478,704 & - & - & - \\
\hline
\end{tabular}

Abbreviation: Mw, molecular weight. 
Table 2 Mean hydrodynamic diameter, polydispersity index, zeta potential, and free thiol of nanoparticles made by different molecular weight of chitosan-GSH

\begin{tabular}{lllll}
\hline Formulation & $\begin{array}{l}\text { Mean hydrodynamic } \\
\text { diameter }(\mathbf{n m})\end{array}$ & $\begin{array}{l}\text { Polydispersity } \\
\text { index }\end{array}$ & $\begin{array}{l}\text { Zeta } \\
\text { potential }\end{array}$ & $\begin{array}{l}\text { Free thiol } \\
(\mu \mathrm{mol} / \mathbf{g})\end{array}$ \\
\hline Chito.I00-GSH-pMMA & $264 \pm 18.1$ & 0.192 & 33.5 & $47.25 \pm 6.04$ \\
Chito.50-GSH-pMMA & $201 \pm 10.2$ & 0.090 & 39.2 & $63.32 \pm 4.86$ \\
Chito.20-GSH-pMMA & $153 \pm 9.0$ & 0.124 & 41.5 & $72.37 \pm 6.32$ \\
\hline
\end{tabular}

Abbreviations: GSH, glutathione; pMMA, poly methyl methacrylate.

sulfhydryl groups of different molecular weights of chitosan is very similar to the results obtained by Bravo-Osuna et al. ${ }^{39}$ The results indicated that when the molecular weight of chitosan increased, the difficulty of the thiol-bearing agent to reach the amino groups of chitosan was increased, and fewer sulfhydryl groups could be fixed on the polymer.

\section{NP preparation and characterization}

The mean hydrodynamic diameter, polydispersity index (PDI), zeta potential, and thiol content of NPs prepared using different molecular weights of chitosan-GSH in a fixed amount of MMA and CAN are presented in Table 2.

The mean size of particles obtained by different molecular weights of thiolated chitosan, with values varying from 153 to $264 \mathrm{~nm}$, demonstrated an incremental increase in accordance with the molecular weight of chitosan. This result is very similar to the results obtained by Bravo-Osuna et al. ${ }^{39}$ The zeta potential of particles was positive, indicating that the negative surface charge of noncoated $\mathrm{pMMA}^{40}$ was completely covered by the cationic chitosan. According to the significant effect of particle size and type of surface coating on the cellular uptake of polymeric $\mathrm{NPs}^{41}$ in this study, chitosan with low molecular weight $(20,000)$ was used for preparation of DTX NPs. Smallest NPs with most free thiol on their surface were produced using Chito.20.

\section{DTX-loaded NPs characterization}

The size, PDI, and zeta potential of DTX-loaded NPs prepared by Chito.20-GSH and different amounts of DTX in a fixed amount of MMA and CAN are seen in Table 3. The particle size is a very important parameter for cellular uptake of the NPs. The permeability of the NPs through the intestinal mucosa decreases when the particle size reaches $500 \mathrm{~nm} .{ }^{41-43}$ The mean diameter of the NPs in this study was $168-186 \mathrm{~nm}$, which is suitable for intestinal uptake of NPs.

As can be seen in Table 3, the zeta potential of the NPs was positive due to their chitosan coating with free amino groups. ${ }^{33}$ This is favorable for making electrostatic bonds with negatively charged mucosa and preventing elimination through the alimentary canal. There is an inverse relationship between the concentration of entrapped DTX and zeta potential value of particles. The SEM image of the NPs shows that they are spherical (Figure 1). The figure also confirms the size of the NPs measured by Zetasizer shown in Table 3.

Figure 2 shows the TEM image of DTX-loaded NPs. The core and coat of NPs can be recognized in this figure.

As given in Table 3, all NPs have a desired PDI less than 0.2 indicating a homogenous size distribution. Commonly, the PDI is an index of stability of all the nanosuspensions.

\section{Entrapment efficiency and drug loading of NPs}

The EE and DL of the NPs is shown in Table 3. The EE of the NPs was determined with an indirect method after isolation of NPs by centrifuging them as described above. Ideally, a successful nanoparticulate system should have a high DL capacity. As seen in Table 3, the EE of Chito.20-GSH-DTX $1 \%, 2 \%, 3 \%$, and $4 \%$ NPs are $92.4,91.1,88.9$, and $93.6 \%$, respectively. The maximum EE was related to NPs with 4\% DTX. This high EE might be due to the tendency of DTX as a hydrophobic molecule to enter the hydrophobic core (pMMA) of the NPs.

Table 3 Characterization of nanoparticles made by Chito. 20 and varying concentrations of DTX

\begin{tabular}{lllllll}
\hline Formulation & DTX $(\mathbf{m g})$ & Size & PDI & $\begin{array}{l}\text { Zeta } \\
\text { potential }\end{array}$ & $\begin{array}{l}\text { Loading } \\
\text { efficiency }\end{array}$ \\
\hline Chito.20-GSH-DTX 1\% & 2.72 & $168 \pm 4.0$ & 0.07 & $36.9 \pm 0.4$ & $8.5 \%$ & $\begin{array}{l}\text { EE } \\
\text { Chito.20-GSH-DTX 2\% }\end{array}$ \\
Chito.20-GSH-DTX 3\% & 5.45 & $174 \pm 5.5$ & 0.10 & $36.2 \pm 0.8$ & $10.34 \%$ & $91.04 \%$ \\
Chito.20-GSH-DTX 4\% & 8.17 & $184 \pm 6.0$ & 0.04 & $35 \pm 0.3$ & $14.5 \%$ & $88.89 \%$ \\
\hline
\end{tabular}

Abbreviations: DTX, docetaxel; EE, entrapment efficiency; GSH, glutathione; PDI, polydispersity index. 


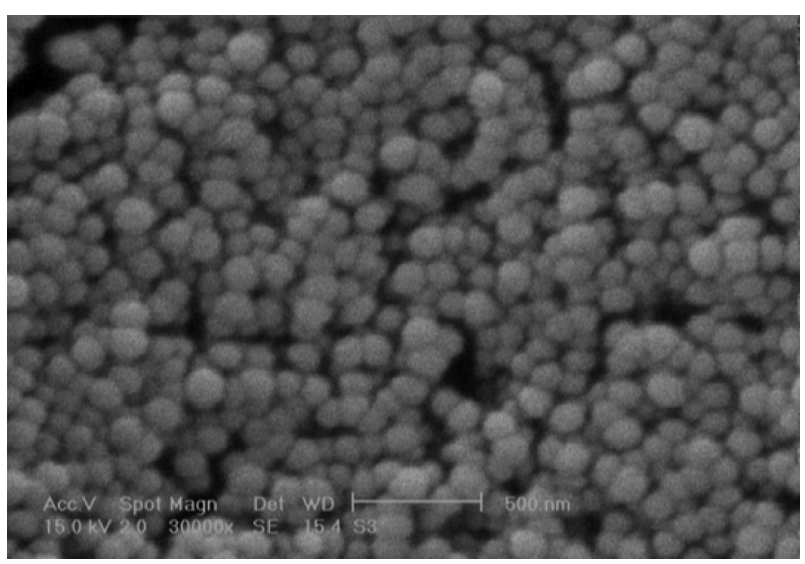

Figure I Scanning electron micrograph of docetaxel-loaded nanoparticles.

The DL of Chito.20-DTX NPs was between $8.5 \%$ and $20.4 \%$. A high DL capacity was expected, due to the hydrophobicity of DTX.

\section{Physical status of DTX in the NPs}

DSC studies were performed to investigate the physical status of DTX in the NPs. Figure 3 shows the DSC thermograms of free DTX, unloaded, and drug-loaded NPs. Free DTX showed a sharp endothermic melting peak at about $169^{\circ} \mathrm{C}$ related to DTX. However, no melting peak could be detected for the NP formulations. This shows that DTX in the NPs is in an amorphous or disordered crystalline phase, miscible in polymeric NPs.

\section{In vitro drug release study}

The in vitro release profile of the DTX-loaded NPs is shown in Figure 4. It shows a sustained Fickian release profile, suggesting that the hydrophobic core acts as a barrier against the release of entrapped DTX from the polymeric matrix into the aqueous solution.

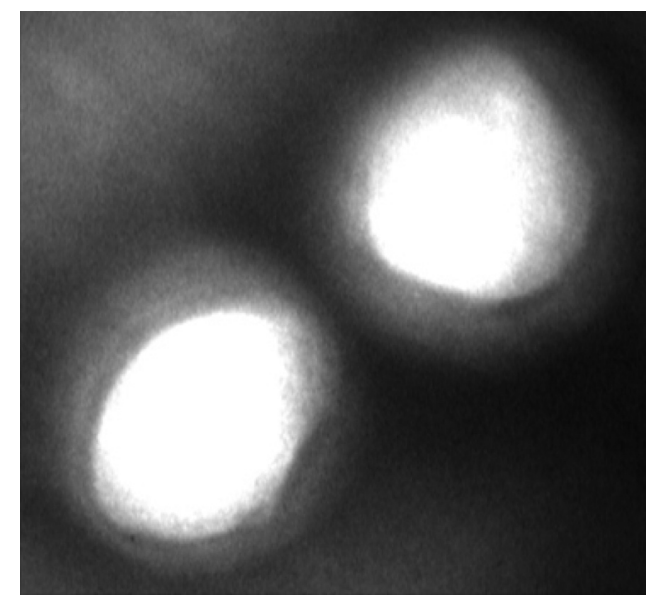

Figure 2 Transmission electron micrograph of a docetaxel-loaded nanoparticle.

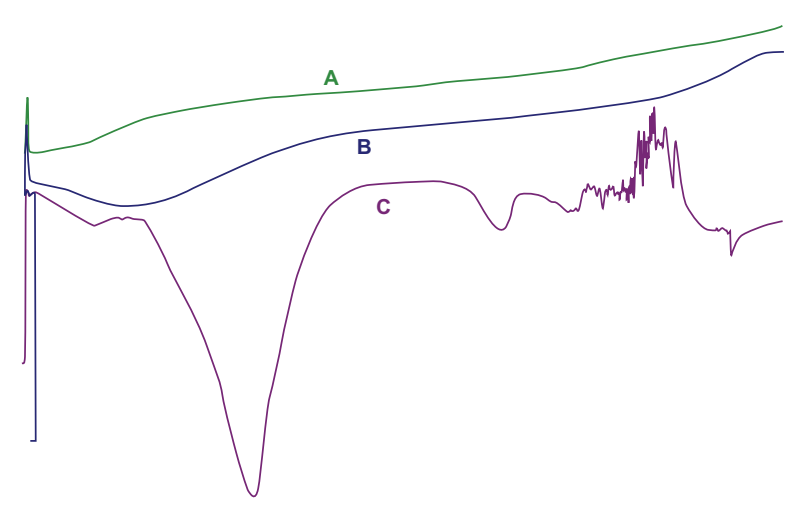

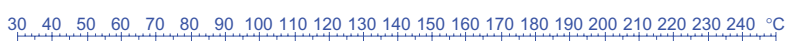
$\begin{array}{llllllllllllllllllllllllll}0 & 2 & 4 & 6 & 8 & 10 & 12 & 14 & 16 & 18 & 20 & 22 & 24 & 26 & 28 & 30 & 32 & 34 & 36 & 38 & 40 & 42 & \mathrm{~nm}\end{array}$

Figure 3 Differential scanning calorimetry thermograms of the docetaxel (DTX)loaded Chito.20 nanoparticles (A), blank Chito.20 nanoparticles (B), and pure $\operatorname{DTX}(\mathrm{C})$.

\section{Fluorescent microscopy image}

Figure 5 shows the fluorescent microscopy image of Caco-2 cells after 2 hours incubation with FA-loaded NPs. As can be seen in this figure, after 2 hours incubation, the FA-loaded NPs have been internalized by the Caco-2 cells, which are located in the cytoplasm around the nucleus.

\section{In vitro cell viability of NPs}

Figure 6 shows the viability of Caco- 2 and MCF- 7 cells after 72 hours incubation time with DTX-loaded NPs in comparison with free DTX drug at the same concentration $(0.1$, $1.0,10,100$, and $1000 \mathrm{ng} / \mathrm{mL}$ ). In our previous study, it was shown that the blank NPs did not show any cytotoxic effect on the Caco- 2 and MCF-7 cells. ${ }^{44}$ The results in this study indicated that the drug entrapped in thiolated Chito.20 NPs had more cytotoxic effect than the free drug for Caco-2 cells at most concentrations $(P<0.05)$ and had equal cytotoxicity for MCF-7 after 72 hours. Based on the result of in vitro release studies (Figure 4), after 72 hours, approximately $70 \%$ of the drug is released from the NPs. Thus, more cytotoxicity effect of the NPs is not only related to the DTX released from

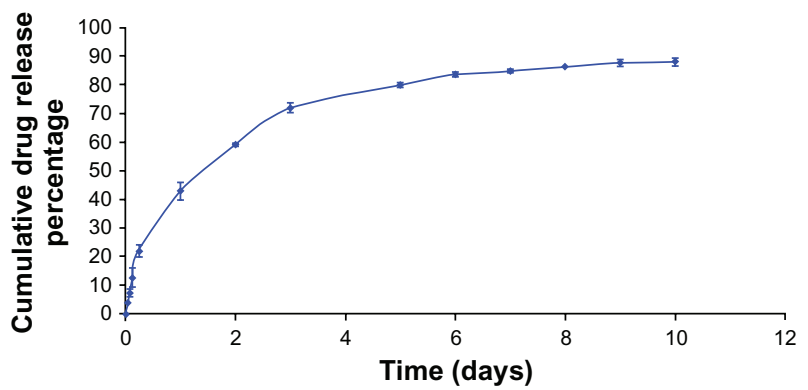

Figure 4 In vitro docetaxel (DTX) release profiles from DTX-loaded nanoparticles in phosphate-buffered solution $\left(\mathrm{pH}=7.4,37^{\circ} \mathrm{C}\right)$. Data are represented as mean \pm standard deviation $(n=3)$. 


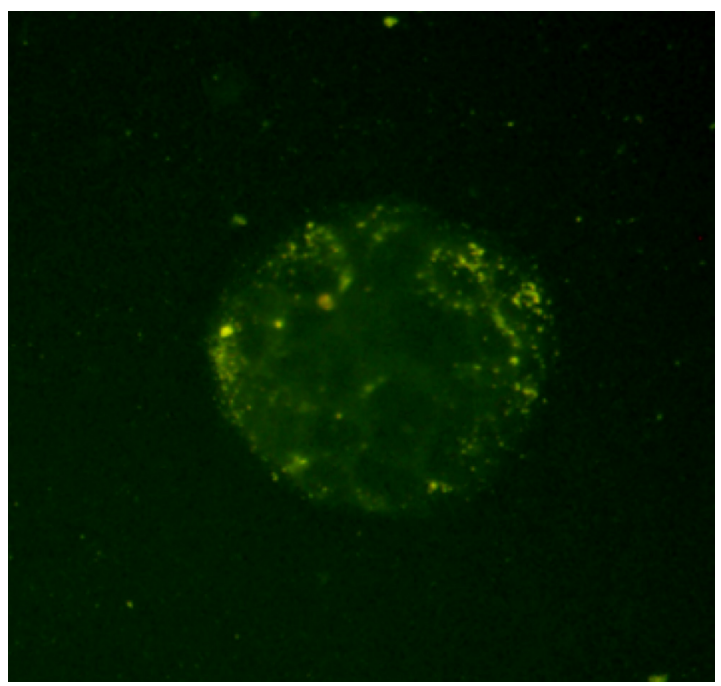

Figure 5 Fluorescence microscopic images of Caco-2 cells after 2 hours incubation with fluoresceinamine (FA)-loaded nanoparticles at $37^{\circ} \mathrm{C}$. As shown in the image, the yellow fluorescence of the FA-loaded nanoparticles is distributed in the cytoplasm, indicating the uptake of nanoparticles by the cells.

\section{A}

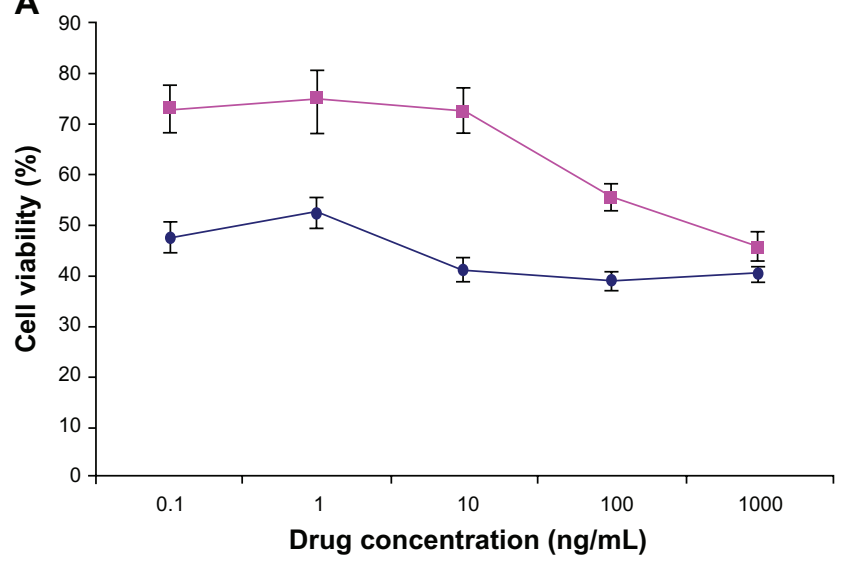

B

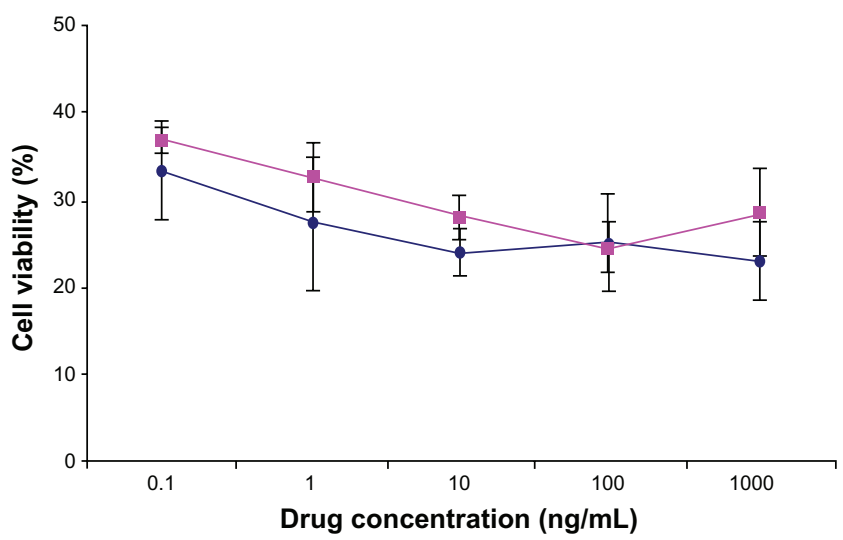

Figure 6 Viability of Caco-2 cells (A) and MCF-7 (B) after 72 hours cell culture with DTX-loaded Chito.20-GSH nanoparticles ( $\bullet$ in comparison with DTX ( $\square)$ at different concentrations $(n=6)$. Percentage survival was assessed by MTT assay. Data are mean \pm standard deviation $(n=6)$.

Abbreviations: DTX, docetaxel; GSH, glutathione; MTT, 3-(4,5-dimethylthiazol-2yl)-2,5-diphenyl tetrazolium bromide).

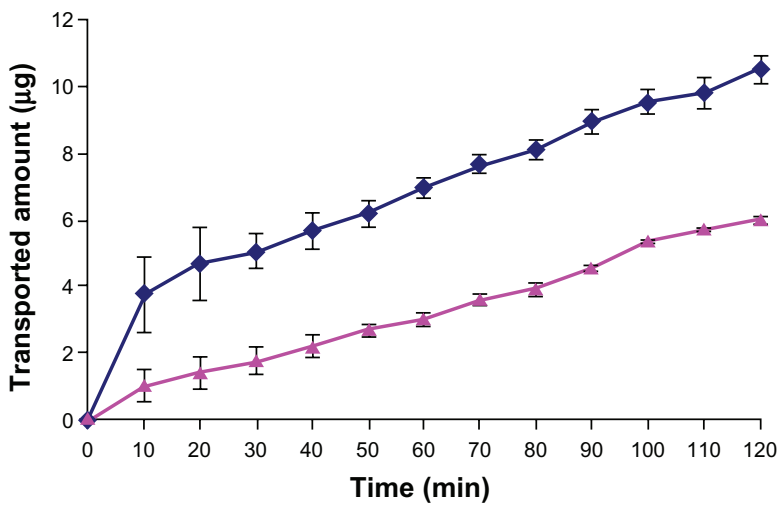

Figure 7 Profile of the amount of DTX transported in medium $(\mathrm{pH} 7.4)$ as a function of time: Chito.20 GSH nanoparticles $(\bullet)$, DTX $(\boldsymbol{\Delta})$. Experiments were carried out in triplicate $(n=3)$.

Abbreviations: DTX, docetaxel; GSH, glutathione.

the NPs but probably also because of cell uptake of the NPs and/or better penetration of NPs into the cells. The site of action of DTX is the cytoplasm, and NPs act as intracellular depots that slowly release the loaded therapeutic agent into the cellular cytoplasm. This increase of cytotoxic effects of NPs after a period of time is in agreement with previous reports. ${ }^{45,46}$

\section{Ex vivo study}

In this study, we used the everted intestinal sac method for measuring the transporting of DTX from the intestinal barrier. Figure 7 shows the amount of DTX transported across the intestinal barrier. As seen in the figure, after 120 minutes, the amount of DTX transported from the intestinal barrier with Chito.20-GSH NPs was significantly higher than free DTX. Consequently, on the basis of these results, it was hypothesized that transportation of DTX across the intestine membrane is low, and the mucoadhesive NPs can increase DTX transport by opening tight junctions and bypassing the efflux pump of p-gp.

\section{Conclusion}

In this work we applied mucoadhesive thiolated chitosan pMMA NPs as a carrier for oral delivery of DTX. DTX was loaded into the pMMA thiolated chitosan NPs using a modified radical polymerization method. This method has some advantages for obtaining a drug carrier, such as elimination of organic solvent and surfactants and preparation of NPs under mild conditions. The size of the NPs was small and size distribution was narrow. EE was high (around 90\%), and in vitro release studies showed the potential of NPs for extended release of DTX in medium solution. It seems 
that the penetration ability of DTX-loaded NPs prepared with thiolated chitosan into the cancer cells causes more cytotoxic effect of NPs versus free drug after 72 hours. Such hydrophobic and mucoadhesive NPs seem to be very promising vehicles for oral delivery of hydrophobic drugs such as taxanes.

\section{Acknowledgments}

The authors would like to thank Nanotechnology Research Centre of Tehran University of Medical Sciences. The authors are grateful to Dr A Kebriaeezadeh, managing director of OSVAH pharmaceutical company, for his support and encouragement of this study, and also thank Mrs S Tavajjohi for her kind assistance in cell culture experiments.

\section{Disclosure}

The authors report no conflicts of interest in this work.

\section{References}

1. Ajani JA, Takiuchi H. Recent developments in oral chemotherapy options for gastric carcinoma. Drugs. 1999;58 Suppl 3:85-90.

2. Feng SS, Dong Y. Poly(D,L-lactide-co-glycolide)/montmorillonite nanoparticles for oral delivery ofanticancer drugs. Biomaterials. 2005;26:6068-6076.

3. Arangoa MA, Ponchel G, Orecchioni AM, Renedo MJ, Duchene D, Irache JM. Bioadhesive potential of gliadin nanoparticulate systems. Eur J Pharm Sci. 2000;11:333-341.

4. Arechabala B, Coiffard C, Rivalland P, Coiffard LJM, de RoeckHoltzhauer Y. Comparison of cytotoxicity of various surfactants tested on normal human fibroblast cultures using the neutral red test, MTT assay and LDH release. J Appl Toxicol. 1999;19:163-165.

5. Atyabi F, Aghaei Moghaddam F, Dinarvand R, Zohuriaan-Mehr MJ, Ponchel G. Thiolated chitosan coated poly hydroxyethyl methacrylate nanoparticles: synthesis and characterization. Carbohydr Polym. 2008;74:59-67.

6. Bernkop-Schnürch A, Kast CE. Chemically modified chitosans as enzyme inhibitors. Adv Drug Deliv Rev. 2001;52:127-137.

7. Mangatal L, Adeline MT, Guénard D, Guéritte-Voegelein F, Potier P. Application of the vicinal oxymination reaction with asymmetric induction to the hemisynthesis of taxol and analogues. Tetrahedron. 1989;45:4177-4190.

8. Kelland LR, Abel G. Comparative in vitro cytotoxicity of taxol and Taxotere against cisplatin-sensitive and -resistant human ovarian carcinoma cell lines. Cancer Chemother Pharmacol. 1992;30:444-450.

9. Eiermann W. Docetaxel - maximising outcomes towards cure in early breast cancer. Breast. 2006;15 Suppl 3:S13-S16.

10. Escobar PF, Rose PG. Docetaxel in ovarian cancer. Expert Opin Pharmacother. 2005;6(15):2719-2726.

11. Iwao-Koizumi K, Matoba R, Ueno N, et al. Prediction of docetaxel response in human breast cancer by gene expression profiling. $J$ Clin Oncol. 2005;23(3):422-431.

12. Van Zuylen L, Verweij J, Sparreboom A. Role of formulation vehicles in taxane pharmacology. Invest New Drugs. 2001;19(2):125-141.

13. Shou M, Martinet M, Korzekwa KR, Krausz KW, Gonzalez FJ, Gelboin HV. Role of human cytochrome P450 3 A4 and 3 A5 in the metabolism of taxotere and its derivatives: enzyme specificity, interindividual distribution and metabolic contribution in human liver. Pharmacogenetics. 1998;8(5):391-401.
14. Malingre MM, Beijnen JH, Schellens JHM. Oral delivery of taxanes. Invest New Drugs. 2001;19(2):155-162.

15. Peracchia MT, Vauthier C, Passirani C, Couvreur P, Labarre D. Complement consumption by poly(ethylene glycol) in different conformations chemically coupled to poly(isobutyl 2-cyanoacrylate) nanoparticles. Life Sci. 1997;61(7):749-761.

16. Lemarchand C, Gref R, Couvreur P. Polysaccharide-decorated nanoparticles. Eur J Pharm Biopharm. 2004;58:327-341.

17. Bravo-Osuna I, Schmitz T, Bernkop-Schnürch A, Vauthier C, Ponchel G. Elaboration and characterization of thiolated chitosan-coated acrylic nanoparticles. Int J Pharm. 2006;316:170-175.

18. Chauvierre C, Labarre D, Couvreur P, Vauthier C. Novel polysaccharide-decorated poly(isobutyl cianoacrylate) nanoparticles. Pharm Res. 2003;20:1786-1793.

19. Chauvierre C, Labarre D, Couvreur P, Vauthier C. Radical polymerization of alkylcyanoacrylates initiated by the redox system dextrancerium (IV) under acidic aqueous conditions. Macromolecules. 2003;36:6018-6027.

20. Hassan EE, Gallo JM. A simple rheological method for the in vitro assessment of mucin-polymer bioadhesive bond strength. Pharm Res. 1990; 7:491-495.

21. Roldo M, Hornof M, Caliceti P, Bernkop-Schnürch A. Mucoadhesive thiolated chitosans as platforms for oral controlled drug delivery: synthesis and in vitro evaluation. Eur J Pharm Biopharm. 2004;57(1):115-121.

22. Leitner VM, Guggi D, Krauland AH, Bernkop-Schnürch A. Nasal delivery of human growth hormone: in vitro and in vivo evaluation of a thiomer/glutathione microparticulate delivery system. J Control Release. 2004;100:87-95.

23. Bernkop-Schnürch A, Kast CE, Guggi D. Permeation enhancing polymers in oral delivery of hydrophilic macromolecules: thiomer/GSH systems. J Control Release. 2003;93:95-103.

24. Bravo-Osuna I, Teutonico D, Arpicco S, Vauthier C, Ponchel G. Characterization of chitosan thiolation and application to thiol quantification onto nanoparticle surface. Int J Pharm. 2007;340(1-2): 173-181.

25. Cui F, Qian F, Yin C. Preparation and characterization of mucoadhesive polymer-coated nanoparticles. Int J Pharm. 2006;316:154-161.

26. Huang M, Khor E, Lim LY. Uptake and cytotoxicity of chitosan molecules and nanoparticles: effects of molecular weight and degree of deacetylation. Pharm Res. 2004;21(2):344-356.

27. Kafedjiiski K, Föger F, Werle M, Bernkop-Schnürchrch A. Synthesis and in vitro evaluation of a novel chitosan-glutathione conjugate. Pharm Res. 2005;22(9):1480-1488.

28. Guggi D, Bernkop-Schnürch A. Improved paracellular uptake by the combination of different types of permeation enhancers. Int J Pharm. 2005;288:141-150.

29. Bernkop-Schnürch A, Schwarz V, Steininger S. Polymers with thiol groups: a new generation of mucoadhesive polymers. Pharm Res. 1999; 16:876-881.

30. Kast CE, Bernkop-Schnürch A. Thiolated polymersthiomers: development and in vitro evaluation of chitosan-thioglycolic acid conjugates. Biomaterials. 2001;22:2345-2352.

31. Cheon Lee S, Kim C, Chan Kwon I, Chung H, Young Jeong S. Polymeric micelles of poly(2-ethyl-2-oxazoline)-block-poly([var epsilon]-caprolactone) copolymer as a carrier for paclitaxel. J Control Rel. 2003;89(3):437-446.

32. Barr WH, Riegelman S. Intestinal drug absorption and metabolism. I. Comparison of methods and models to study physiological factors of in vitro and in vivo intestinal absorption. J Pharm Sci. 1970;59(2): 154-163.

33. Bravo-Osuna I, Ponchel G, Vauthier C. Tuning of shell and core characteristics of chitosan-decorated acrylic nanoparticles. Eur J Pharm Sci. 2007;30(2):143-154.

34. Chen M-C, Wong H-S, Lin K-J, et al. The characteristics, biodistribution and bioavailability of a chitosan-based nanoparticulate system for the oral delivery of heparin. Biomaterials. 2009;30(34):6629-6637. 
35. Vila A, Sanchez A, Janes K, et al. Low molecular weight chitosan nanoparticles as new carriers for nasal vaccine delivery in mice. Eur $J$ Pharm Biopharm. 2004;57(1):123-131.

36. Lin YH, Chen CT, Liang HF, et al. Novel nanoparticles for oral insulin delivery via the paracellular pathway. Nanotechnology. 2007; 18(10):1-11.

37. Janes KA, Alonso MJ. Depolymerized chitosan nanoparticles for protein delivery: preparation and characterization. J Appli Polym Sci. 2003;88(12):2769-2776.

38. Mao S, Shuai X, Unger F, Simon M, Bi D, Kissel T. The depolymerization of chitosan: effects on physicochemical and biological properties. Int J Pharm. 2004;281(1-2):45-54.

39. Bravo-Osuna I, Ponchel G, Vauthier C. Tuning of shell and core characteristics of chitosan-decorated acrylic nanoparticles. Eur J Pharm Sci. 2007;30:143-154.

40. Passirani C, Barratt G, Devissaguet J-P, Labarre D. Interactions of nanoparticles bearing heparin or dextran covalently bound to poly(methyl methacrylate) with the complement system. Life Sci. 1998;62(8):775-785.
41. Feng SS, Wina KY. Effects of particle size and surface coating on cellular uptake of polymeric nanoparticles for oral delivery of anticancer drugs. Biomaterials. 2005;26:2713-2722.

42. Florence AT. Nanoparticle uptake by the oral route: fulfilling its potential? Drug Discov Today Technol. 2005;2(1):75-81.

43. Norris DA, Sinko PJ. Effect of size, surface charge, and hydrophobicity on the translocation of polystyrene microspheres through gastrointestinal mucin. J Appl Polym Sci. 1997;63:1481-1492.

44. Akhlaghi SP, Saremi S, Ostad SN, Dinarvand R, Atyabi F. Discriminated effects of thiolated chitosan-coated pMMA paclitaxel-loaded nanoparticles on different normal and cancer cell lines. Nanomedicine. 2010;6(5):689-697.

45. Esmaeili F, Dinarvand R, Ghahremani MH, Ostad SN, Esmaily H, Atyabi F. Cellular cytotoxicity and in-vivo biodistribution of docetaxel poly(lactide-co-glycolide) nanoparticles. Anticancer Drugs. 2010;21: 43-52.

46. Zhao J, Kim JE, Reed E, Li QQ. Molecular mechanism of antitumor activity of taxanes in lung cancer. Int J Oncol. 2005;27(247-256).
International Journal of Nanomedicine

\section{Publish your work in this journal}

The International Journal of Nanomedicine is an international, peerreviewed journal focusing on the application of nanotechnology in diagnostics, therapeutics, and drug delivery systems throughout the biomedical field. This journal is indexed on PubMed Central, MedLine, CAS, SciSearch $\AA$, Current Contents ${ }^{\circledR} /$ Clinical Medicine,

\section{Dovepress}

Journal Citation Reports/Science Edition, EMBase, Scopus and the Elsevier Bibliographic databases. The manuscript management system is completely online and includes a very quick and fair peer-review system, which is all easy to use. Visit http://www.dovepress.com/ testimonials.php to read real quotes from published authors. 